\title{
Ouverture de la PMA aux couples de femmes : une (r)évolution qui s'impose
}

\begin{abstract}
Alors que le projet de mariage pour tous et toutes semble sur le point d'être accepté par les Chambres fédérales, la question de l'accès à la procréation médicalement assistée (PMA) pour les couples de femmes est, quant à elle, beaucoup plus controversée. Dans cette contribution, l'auteure examine dans quelle mesure l'exclusion des couples de femmes des techniques de procréation médicalement assistée est conforme au principe de non-discrimination consacré par la CEDH.
\end{abstract}

Catégories d'articles: Contributions

Domaines juridiques : Droits fondamentaux ; Droit de la famille. Droit du mariage 


\section{Table des matières}

1. Introduction

2. Cadre constitutionnel

3. Examen de la conventionnalité de la loi sur la procréation médicalement assistée

3.1. La loi sur la procréation médicalement assistée

3.2. La jurisprudence de la Cour européenne des droits de l'homme

3.3. Examen critique

3.3.1. Traitement comparable de situations différentes

3.3.2. Motifs justificatifs

4. Projet de mariage pour tous et toutes

5. Conclusion

\section{Introduction}

[1] Alors qu'un projet de mariage pour tous et toutes est actuellement pendant devant les Chambres fédérales et semble particulièrement bien soutenu par la population ${ }^{1}$, la question de l'accès à la procréation médicalement assistée (PMA) pour les couples lesbiens semble, quant à elle, plus controversée. À l'instar de la majorité des membres de la Commission des affaires juridiques du Conseil national, le Conseil fédéral s'est opposé au traitement de cette question dans le cadre du projet de mariage pour tous et toutes ${ }^{2}$. Et vingt-deux cantons ont soutenu une approche comparable dans le cadre de la procédure de consultation ${ }^{3}$.

[2] Cette contribution vise à examiner dans quelle mesure l'exclusion des couples de femmes de tout accès à la PMA est conforme aux exigences de la jurisprudence de la Cour européenne des droits de l'homme. Notre analyse commencera par un examen de la législation en vigueur, puis se penchera, dans un second temps, sur le projet pendant devant les Chambres fédérales.

\section{Cadre constitutionnel}

[3] À titre liminaire, il y a lieu de rappeler le cadre constitutionnel applicable. Se pose en effet la question de savoir dans quelle mesure l'exclusion des couples de femmes se déduit directement de la Constitution fédérale (Cst. féd.).

[4] Conformément à l'art. 119 al. 2 let. c Cst. féd., « le recours aux méthodes de procréation médicalement assistée n'est autorisé que lorsque la stérilité ou le danger de transmission d'une grave maladie ne peuvent être écartés d'une autre manière, et non pour développer chez l'enfant certaines qualités ou pour faire de la recherche $»^{4}$. Au vu du texte de cette disposition se pose la question de l'interprétation à donner à la notion de stérilité. Exclut-elle par essence les couples de même sexe ou peut-elle être interprétée plus largement?

1 Chams Laz, Plus de 80 \% des Suisses sont favorables au mariage pour les couples de même sexe, Le Temps, 10 février 2020.

2 Avis du Conseil fédéral du 29 janvier 2020, Initiative parlementaire " Mariage civil pour tous », FF 20201223 ss.

3 Rapport de la Commission des affaires juridiques du Conseil national du 30 août 2019, Initiative parlementaire « Mariage civil pour tous », FF 20201224 note 2.

4 Ces conditions sont reprises par l’art. 5 de la loi fédérale sur la procréation médicalement assistée du 18 décembre 1998 (LPMA), RS 810.11. 
[5] Dans son message relatif à la loi sur le partenariat enregistré entre personnes du même sexe (LPart), le Conseil fédéral relevait qu'il y a "stérilité lorsqu'aucune grossesse n'intervient, malgré des rapports réguliers et non protégés, durant une ou deux années $»^{5}$. Le Conseil fédéral se référait à l'aspect biologique de l'infertilité ${ }^{6}$ et excluait donc les couples de même sexe ${ }^{7}$. Une telle interprétation n'est toutefois plus suivie par la doctrine majoritaire. Comme l'a rappelé ZiegLer, cette interprétation restrictive de la stérilité se déduit d'une définition qui a été donnée par l'Organisation mondiale de la santé (OMS) dans une situation précise : il s'agissait, pour l'OMS, de définir le moment à partir duquel un couple hétérosexuel peut être qualifié de stérile ${ }^{8}$. Or il est tout à fait envisageable d'appréhender la notion de stérilité de façon moins hétéronormative : comme le relève la Commission nationale d'éthique, la notion de stérilité peut en effet être interprétée plus largement, en se référant, par exemple, aux types de liens affectifs - ce qui permet d'inclure les couples de même sexe - ou à l'absence de tels liens - ce qui permet d'inclure les personnes seules ${ }^{9}$.

[6] Avec la doctrine majoritaire, nous sommes d'avis que la notion de stérilité ne doit pas être lue dans un sens biologique strict, mais doit au contraire être appréhendée largement pour inclure tout désir non réalisé d'enfant ${ }^{10}$. Une telle interprétation s'impose au regard de la Convention européenne des droits de l'homme. L'approche biologique entraînerait en effet une différence de traitement fondée sur l'orientation sexuelle ${ }^{11}$ qui n'est pas susceptible de se justifier - nonobstant l'avis du Conseil fédéral - par l'objectif de "garantir l'ordre naturel des choses ${ }^{12}$, la PMA entraînant précisément « un éclatement du cadre naturel de la procréation ${ }^{13}$.

5 Message du 26 juin 1996 relatif à l'initiative populaire « pour la protection de l'être humain contre les techniques de reproduction artificielle (Initiative pour une procréation respectant la dignité humaine, PPD) » et à la loi fédérale sur la procréation médicalement assistée (LPMA), FF 1996 III 197.

6 Commission nationale d'éthique, La procréation médicalement assistée. Considérations éthiques et propositions pour l'avenir, Prise de position $n^{\circ} 22 / 2013$, 39; Véronique BoIllet, in : Commentaire romand de la Constitution fédérale, Art. 119 N 37 ss, à paraître.

7 Dans le même sens, Jean-François Aubert, in : Petit commentaire de la Constitution fédérale de la Confédération suisse du 18 avril 1999, Zurich/Bâle/Genève 2003, art. 119 N 19 ; Ruth Reusser/Rainer J. Schweizer, in : St. Galler Kommentar zur schweizerischen Bundesverfassung, Art. 119 N 34.

8 À cet égard, cf. https://www.who.int/reproductivehealth/topics/infertility/multiple-definitions/en/.

9 Commission nationale d'éthique, La procréation médicalement assistée. Considérations éthiques et propositions pour l'avenir, Prise de position $n^{\circ} 22 / 2013,39$.

10 Ivo Hangartner, Verfassungsrechtliche Grundlagen einer registrierten Partnerschaft für gleichgeschlechtliche Paare, PJA 2001, note 44 ; Karin HochL, Gleichheit - Verschiedenheit. Die rechtliche Regelung gleichgeschlechtlicher Partnerschaften in der Schweiz im Verhältnis zur Ehe, Saint-Gall 2002, p. 83 ; JonAs SCHWEIGHAUSER, in : Zürcher Kommentar zum Partnerschaftsgesetz Kommentar zum Bundesgesetz über die eingetragene Partnerschaft gleichgeschlechtlicher Paare (PartG) vom 18. Juni 2004, Art. 28 LPart N 37, Zurich 2007 ; Eva Maria Belser/Eva Molinari, in : Basler Kommentar zur schweizerischen Bundesverfassung, Art. 119 N 30 , Bâle 2015 ; Eva Maria Belser/Alexandra Jungo, Elternschaft im Zeitalter medizinischer Machbarkeit, RDS I 2016, p. 191 ; ANDreas Ziegler, « Ehe für alle » und Fortpflanzungsmedizin in der Schweiz, in : Jusletter 8 avril 2019, Rz 47 ss. Contra : Ruth Reusser/Rainer J. Schweizer, in : St. Galler Kommentar zur schweizerischen Bundesverfassung, Art. 119 N 34, $3^{\text {e }}$ éd., Zurich 2014; Giovanni Biaggini, in : BV Kommentar, Art. 119 N 14, $2^{e}$ éd., Zürich 2017 ; Dевован Staub, Verfassungsrechtlicher Rahmen der Präimplantationsdiagnostikverfahren, Thèse, Zurich/Saint-Gall 2019, p. 10, 12 ss.

11 Comme nous le verrons ensuite, « les différences fondées sur l'orientation sexuelle doivent être justifiées par des raisons particulièrement graves », CourEDH, arrêt Gas et Dubois c. France du 15.3.2012, n² 25951/07, § 59 et les nombreuses références citées.

12 Message du 29 novembre 2002 relatif à la loi fédérale sur le partenariat enregistré entre personnes du même sexe, FF 20031222.

13 Commission nationale d'éthique, La procréation médicalement assistée. Considérations éthiques et propositions pour l'avenir, Prise de position $n^{\circ} 22 / 2013,9$. 
[7] Par comparaison, rappelons que d'autres cadres normatifs n'offrent pas une telle marge d'interprétation, dès lors qu'ils se réfèrent expressément au caractère biologique de la stérilité. Tel est par exemple le cas de la législation française, selon laquelle le "caractère pathologique de l'infertilité doit être médicalement diagnostique ${ }^{14}$. C'est d'ailleurs sur la base du caractère pathologique de l'infertilité que le Conseil d'État a refusé de renvoyer au Conseil constitutionnel la question prioritaire de constitutionnalité invoquée par un couple de femmes ${ }^{15}$, considérant que " les couples formés d'un homme et d'une femme sont, au regard de la procréation, dans une situation différente de celle des couples de personnes de même sexe. Il résulte des dispositions de l'article L. 2141-2 du code de la santé publique qu'en réservant l'accès à l'assistance médicale à la procréation aux couples composés d'un homme et d'une femme, vivants, en âge de procréer et souffrant d'une infertilité médicalement diagnostiquée, le législateur a entendu que l'assistance médicale à la procréation ait pour objet de remédier à l'infertilité pathologique d'un couple sans laquelle celui-ci serait en capacité de procréer. La différence de traitement, résultant des dispositions critiquées, entre les couples formés d'un homme et d'une femme et les couples de personnes de même sexe est en lien direct avec l'objet de la loi qui l'établit et n'est, ainsi, pas contraire au principe d'égalité $»^{16}$.

[8] Si cette référence expresse au caractère pathologique permet de distinguer le cadre normatif suisse du cadre normatif français et peut justifier une interprétation différente de la notion de stérilité, relevons néanmoins que l'analyse du Conseil d'État a fait l'objet de critiques - légitimes selon nous - au sein de la doctrine. Plusieurs auteurs jugent en effet que cette distinction entre, d'une part, la réponse médicale à une pathologie et, d'autre part, les demandes sociétales visant à satisfaire un désir d'enfant n'est pas pertinente dès lors que la PMA avec tiers donneurs " ne guérit pas la stérilité (en rendant les personnes aptes a avoir des enfants), mais la pallie et [...] est en ce sens aussi thérapeutique que l'adoption $»^{17}$. En fin de compte, comme le relève MorTier, la comparaison entre la situation d'un couple de femmes et celle d'un couple hétérosexuel stérile présente « plus de ressemblances que de différences [...] au regard de la procréation : dans tous les cas, ni l'un ni l'autre de ces couples ne parviendra a concevoir un enfant sans l'aide d'un

14 Art. L2141-2 du Code français de santé publique : « L'assistance médicale à la procréation est destinée à répondre à la demande parentale d'un couple. Elle a pour objet de remédier à l'infertilité dont le caractère pathologique a été médicalement diagnostiqué ou d'éviter la transmission à l'enfant ou à un membre du couple d'une maladie d'une particulière gravité. [...]».

15 À noter encore que c'est à l'aune du principe d'égalité - et non à celle de l'interdiction de discrimination - que l'analyse a été effectuée. Sur cette distinction, cf. FERDINAND MéLin-Soucramanien, La loi et le principe d'égalité, RFDA 2013, p. 952 ss.

16 Conseil d'État, $1^{\text {re }}$ et $4^{\mathrm{e}}$ chambres réunies, 28/09/2018, 421899. Voir également Conseil d'État, Révision de la loi de bioéthique : quelles options pour demain?, 28 juin 2018, p. 51 : «Eu égard a la finalité de compensation d'une infertilité pathologique que la loi assigne a la procréation médicalement assistée, une personne seule, un couple homosexuel, un veuf ou une veuve sont placés dans des situations différentes de celle des couples hétérosexuels infertiles puisque l'impossibilité de procréer a laquelle ils sont confrontés ne résulte pas d'une pathologie. Cela ne disqualifie pas pour autant leur projet parental, mais ces catégories de personnes ne peuvent revendiquer un traitement analogue au nom du principe d'égalité ou de nondiscrimination. » Voir encore Conseil Constitutionnel, Décision n² 2013-669 DC du 17 mai 2013 : «Les couples formés d'un homme et d'une femme sont, au regard de la procréation, dans une situation différente de celle des couples de personnes de même sexe; que le principe d'égalité ne s'oppose pas a ce que le législateur règle de façon différente des situations différentes dès lors que la différence de traitement qui en résulte est en lien direct avec l'objet de la loi qui l'établit; que, par suite, [...] le principe d'égalité [...] n'imposa[it] qu'en ouvrant le mariage et l'adoption aux couples de personnes de même sexe, le législateur modifie la législation régissant ces différentes matières. »

17 Recherches et études sur le genre et les inégalités dans les normes en Europe (REGINE), Droit et genre, Recueil Dalloz, 2019, p. 856 ss. Voir également AnAëlle CAPpellari, L'influence du droit de la sante sur le droit extrapatrimonial de la famille - Repenser le droit français a la lumière du droit suisse, Thèse, Neuchâtel 2016, p. 642. 
tiers et sans l'assistance de la médecine ${ }^{18}$. Telle avait d'ailleurs également été l'approche de la Cour constitutionnelle autrichienne qui, en 2013 déjà, avait considéré qu'une femme lesbienne se trouve dans une situation comparable à une femme hétérosexuelle dont le partenaire n'est pas capable de procréer ${ }^{19}$ et avait jugé contraire aux art. 8 et $14 \mathrm{CEDH}$ la législation autrichienne qui excluait les couples de femmes du don de sperme ${ }^{20}$.

\section{Examen de la conventionnalité de la loi sur la procréation médicale- ment assistée}

\subsection{La loi sur la procréation médicalement assistée}

[9] Alors même que la Constitution fédérale n'exclut pas les couples de même sexe du droit d'accéder à la PMA, la loi fédérale sur la procréation médicalement assistée (LPMA) réserve ces techniques « aux couples à l'égard desquels un rapport de filiation peut être établi (au sens des art. 252 à 263 du code civil, CC) ", soit aux seuls couples hétérosexuels (art. 3 al. 2 let. a LPMA). Le don de sperme est quant à lui réservé aux couples hétérosexuels mariés (art. 3 al. 3 LPMA) ${ }^{21}$.

[10] Du fait qu'elle exclut expressément les couples de même sexe, cette réglementation doit être examinée quant à sa conformité à l’art. $14 \mathrm{CEDH}$.

\subsection{La jurisprudence de la Cour européenne des droits de l'homme}

[11] Au préalable, commençons par rappeler que la Cour européenne des droits de l'homme (CourEDH) n'a, à ce jour, pas eu à traiter directement de la question de l'accès des couples de femmes à la PMA. Dans la seule affaire ${ }^{22}$ qui traitait précisément de cette question, la Cour a jugé la requête des deux femmes irrecevable faute d'épuisement des voies de recours internes ${ }^{23}$.

[12] Malgré l'absence d'arrêts portant directement sur la question qui nous occupe, de nombreuses considérations ont été développées par la Cour, s'agissant tant de l'accès à la PMA que de l'interdiction de discrimination fondée sur l'orientation sexuelle.

18 Thomas Dumortier, Le principe d'égalité et l'ouverture de la PMA aux couples de femmes et aux femmes célibataires, RDP 1/2019, p. 136 ss.

19 «Wenn die Minderheit lesbische Frauen auf das Ausland verweist (S. 9), so ist darauf hinzuweisen, dass heterosexuellen Frauen in der gleichen Situation (zeugungsunfähiger Partner) die Vornahme im Ausland mit allen tatsächlichen, rechtlichen und finanziellen Schwierigkeiten eben gerade nicht zugemutet wird ", Verfassungsgerichtshof (VfGH), Erkenntnis 10.12.2013 G 16/2013-16, G 44/2013-14 § 44.

20 Verfassungsgerichtshof (VfGH), Erkenntnis 10.12.2013 G 16/2013-16, G 44/2013-14

21 L'art. 28 LPart prévoit également que «Les personnes liées par un partenariat enregistré ne sont pas autorisées à adopter un enfant conjointement ni à recourir à la procréation médicalement assistée ", Loi fédérale sur le partenariat enregistré entre personnes du même sexe du 18 juin 2004, RS 211.231. CourEDH, Charron et Merle-Montet c. France, 16 janvier 2018, nº 22612/15.

23 À noter néanmoins que dans l'affaire Gas et Dubois c. France, qui visait un refus de l'adoption simple d'un enfant par la femme vivant avec la mère biologique dans le cadre d'un couple homosexuel, la CourEDH a précisé que " [...] si le droit français ne prévoit l'accès à ce dispositif [IAD] que pour les couples hétérosexuels, cet accès est également subordonné a l'existence d'un but thérapeutique, visant notamment à remédier à une infertilité dont le caractère pathologique a été médicalement constaté ou à éviter la transmission d'une maladie grave [...]. Ainsi, pour l'essentiel, l'IAD n'est autorisée en France qu'au profit des couples hétérosexuels infertiles, situation qui n'est pas comparable à celle des requérantes. Il s'ensuit, pour la Cour, que la législation française concernant l'IAD ne peut être considérée comme étant à l'origine d'une différence de traitement dont les requérantes seraient victimes. » 
[13] En ce qui concerne tout d'abord le droit d'accès à la PMA, il y a lieu de rappeler que la CourEDH juge que « le droit des couples à concevoir un enfant et à recourir pour ce faire à la procréation médicalement assistée relève $[. .$.$] de la protection de l'article 8$, pareil choix constituant une forme d'expression de la vie privée et familiale $»^{24}$.

[14] Le droit de recourir à la PMA relevant du champ d'application de l'art. $8 \mathrm{CEDH}$, sa jouissance doit être assurée sans distinction, conformément à l'art. 14 CEDH. Or la Cour a eu l'occasion de rappeler à maintes reprises que pour ne pas être jugées discriminatoires, « les différences fondées sur l'orientation sexuelle doivent être justifiées par des raisons particulièrement graves ${ }^{25}$.

[15] Relevons toutefois que la Cour continue à admettre des différences de traitement lorsqu'elles sont fondées sur le statut marital ${ }^{26}$. Elle a ainsi rappelé, dans son arrêt Gas et Dubois c. France, que « le mariage confère un statut particulier à ceux qui s'y engagent. L'exercice du droit de se marier est protégé par l'article $12 \mathrm{CEDH}$ et emporte des conséquences sociales, personnelles et juridiques ${ }^{27}$. Forte de ce constat, la Cour a jugé que deux femmes liées par un partenariat ne se trouvent pas dans une situation juridique comparable à celle d'un couple hétérosexuel marié en matière d'adoption par le second parent ${ }^{28}$.

[16] En définitive, l'on peut donc constater que si la Cour est stricte en matière de discrimination fondée sur l'orientation sexuelle, elle semble en revanche admettre que la situation juridique des couples de même sexe qui vivent en partenariat ou en concubinage n'est pas comparable à celle des couples mariés et que les différences de traitement entre ces différentes situations ne doivent pas nécessairement être qualifiées de discriminatoires.

\subsection{Examen critique}

[17] Si l'on s'en tient aux arrêts évoqués préalablement, on pourrait défendre que le régime actuellement en vigueur ne pose pas de difficultés au regard de la CEDH. En effet, bien qu'il puisse sembler problématique dès lors qu'il instaure une différence de traitement fondée sur l'orientation sexuelle - la PMA étant ouverte à tous les couples hétérosexuels et fermée à tous les couples de même sexe -, l'art. 3 al. 2 LPMA réserve quant à lui l'accès au don de sperme aux seuls couples hétérosexuels mariés et pourrait, à ce titre, permettre de justifier la différence de traitement puisque celle-ci se fonde sur le statut marital pour exclure les couples de femmes.

[18] Une telle analyse nous semble cependant devoir évoluer au regard de la jurisprudence récente de la CourEDH dont la mobilisation permet de proposer un examen plus critique de la réglementation en vigueur en Suisse.

[19] Il est à cet égard intéressant de se référer à l'arrêt Taddeucci et McCall c. Italie ${ }^{29}$ que la CourEDH a rendu en matière de regroupement familial.

\footnotetext{
24 CourEDH, S. H. c. Autriche, 3 novembre 2011 (GC), n 57813/00, § 82.

25 CourEDH, Gas et Dubois c. France, 15 mars 2012, n 25951/07, § 59.

26 Belser/Jungo (note 10), p. $201 \mathrm{~s}$.

27 CourEDH, Gas et Dubois c. France, 15 mars 2012, n² 25951/07, § 68.

28 CourEDH, Gas et Dubois c. France, 15 mars 2012, n² 25951/07, § 68.

29 CourEDH, Taddeucci et McCall c. Italie, 30 juin 2016, n 51362/09.
} 


\subsubsection{Traitement comparable de situations différentes}

[20] Dans cet arrêt, la CourEDH a examiné une loi italienne qui réservait le droit au regroupement familial aux couples mariés hétérosexuels. La Cour est arrivée à la conclusion qu' « en décidant de traiter, aux fins de l'octroi du permis de séjour pour raison familiale, les couples homosexuels de la même manière que des couples hétérosexuels n'ayant pas régularisé leur situation, l'État a enfreint le droit des requérants de ne pas subir de discrimination fondée sur l'orientation sexuelle dans la jouissance de leurs droits au regard de l'article $8 \mathrm{CEDH} »^{30}$. Selon la Cour, les couples homosexuels ont en effet été traités, en ce qui concerne l'octroi d'un permis de séjour pour raison familiale, de la même façon que des personnes se trouvant dans une situation sensiblement différente de la leur, à savoir des partenaires hétérosexuels ayant décidé de ne pas régulariser leur situation. Or, « c'est précisément l'absence de la possibilité, pour les couples homosexuels, d'avoir accès à une forme de reconnaissance légale qui a placé les requérants dans une situation différente de celle d'un couple hétérosexuel non marié »" ${ }^{31}$. Cette jurisprudence est, à notre sens, fondamentale, dans la mesure où elle ne permet plus de refuser l'examen du caractère discriminatoire d'une réglementation par la seule référence au statut marital. En effet, bien au contraire, la CourEDH rappelle ici que si un État refuse d'ouvrir l'institution du mariage aux couples de même sexe, il n'est alors pas possible de traiter ces couples de manière comparable aux couples hétérosexuels ayant renoncé à se marier.

[21] Si l'on transpose cette jurisprudence à la question de l'accès au don de sperme, il nous paraît tout à fait admissible de soutenir que les couples de femmes ont été traités, en ce qui concerne cet accès au sens de l'art. 3 al. 3 LPMA, de la même façon que des couples se trouvant dans une situation sensiblement différente de la leur, à savoir des concubins hétérosexuels ayant décidé de ne pas se marier - qui sont, pour rappel, également exclus du don selon l'art. 3 al. 3 LPMA. En effet, le fait de réserver l'accès au don de sperme aux seuls couples mariés exclut de manière indirecte les couples de même sexe du bénéfice de ce droit (comme c'était le cas en Italie s'agissant du droit au regroupement familial) dès lors que ces derniers n'ont pas accès au mariage en Suisse. En d'autres termes, l'exigence du statut marital consacrée par l'art. 3 al. 3 LPMA « ne constitue un obstacle insurmontable [pour l'accès au don de sperme] que pour les couples homosexuels $»^{32}$.

\subsubsection{Motifs justificatifs}

[22] L'examen de la conformité de l'art. 3 al. 3 LPMA à l'art. 14 CEDH n'en est pas pour autant terminé. Il reste à déterminer si le fait que la LPMA ne prévoit pas de traitement différencié à l'égard des couples de même sexe est susceptible de se justifier. Dans ce même arrêt, la Cour rappelle " qu'une différence de traitement de situations analogues ou un traitement comparable de situations différentes sont discriminatoires s'ils ne reposent pas sur une justification objective et raisonnable, c'est-à-dire s'ils ne poursuivent pas un but légitime ou s'il n'y a pas un rapport raisonnable de proportionnalité entre les moyens employés et le but visé » ${ }^{33}$. En l'espèce, la Cour juge que si la protection de la famille traditionnelle peut, dans certaines circonstances, constituer

30 CourEDH, Taddeucci et McCall c. Italie, 30 juin 2016, $n^{\circ} 51362 / 09$, § 98.

31 CourEDH, Taddeucci et McCall c. Italie, 30 juin 2016, n 51362/09, § 95.

32 CourEDH, Taddeucci et McCall c. Italie, 30 juin 2016, n 51362/09, § 83.

33 CourEDH, Taddeucci et McCall c. Italie, 30 juin 2016, $n^{\circ} 51362 / 09, \S 87$. 
un motif objectif admissible, elle ne peut être admise lorsqu'il est question de l'octroi d'un permis de séjour pour raison familiale à un partenaire étranger homosexuel, car elle ne représente pas une raison " particulièrement solide et convaincante ${ }^{34}$.

[23] Qu'en est-il dès lors des motifs susceptibles de justifier l'exclusion des couples de femmes du don de sperme? Dans un arrêt ancien de 1989, le Tribunal fédéral a jugé qu'il était admissible de limiter le don de sperme aux couples hétérosexuels mariés (par opposition aux concubins hétérosexuels, aucune référence n'étant faite aux couples de même sexe) au nom du bien de l'enfant et de l'intérêt public de limiter le nombre d'enfants privés de père - évoquant les difficultés liées à l'établissement du lien de filiation à l'égard d'un père social qui ne serait pas marié à la mère ${ }^{35}$. Le Conseil fédéral a, quant à lui, relevé dans son message relatif à la LPMA que " [1]a nature veut que chaque enfant ait un père et une mère. Ces personnes ont une importance spécifique pour le développement de l'enfant et sont en général considérées juridiquement comme ses parents. Ces principes fondamentaux de la nature humaine doivent être respectés lors de la mise en œuvre de la PMA. Ainsi, ces techniques doivent être envisagées uniquement pour des couples hétérosexuels, dont les membres entendent assumer ensemble la responsabilité parentale de l'enfant ${ }^{36}$. S'agissant plus particulièrement de l'exigence du mariage pour l'accès au don de sperme, le Conseil fédéral faisait valoir qu'elle seule permettait de garantir la stabilité nécessaire au bien de l'enfant ${ }^{37}$. Relevons toutefois que le Conseil fédéral se limitait ici à examiner la question de l'exclusion des couples hétérosexuels non mariés (et ne traitait pas la question de l'exclusion des couples de femmes ou des femmes seules). En définitive, c'est tant la nature que l'intérêt de l'enfant qui sont invoqués pour justifier l'exclusion des couples de femmes du don de sperme. Or au regard des objectifs qu'elles sont censées viser, de telles raisons ne sont, à notre sens, pas suffisamment solides et convaincantes.

[24] S'agissant tout d'abord du critère de la nature, il n'est, comme déjà évoqué, absolument pas pertinent dès lors que la PMA implique précisément « un éclatement du cadre naturel de la procréation $»^{38}$. Un tel argument fait en effet référence à la situation d'un couple hétérosexuel fertile composé d'un homme et d'une femme ayant la capacité d'engendrer. Or un tel couple ne nécessite précisément pas une PMA avec don de sperme ${ }^{39}$. À cela s'ajoute que l'argument selon lequel "la nature veut que chaque enfant ait un père et une mère " n'est pas non plus pertinent dès lors que, comme on le verra, l'ouverture du don de sperme aux couples de femmes permettra justement aux enfants d'avoir accès à leurs origines et d'être informés sur leur ascendance paternelle (cf. § 32). Pour le surplus, un tel argument ne peut par ailleurs plus être défendu d'un point de vue systématique dès lors que le législateur a admis qu'une personne puisse adopter l'enfant de

34 CourEDH, Taddeucci et McCall c. Italie, 30 juin 2016, n 51362/09, § 93.

35 ATF 115 Ia 234 c. 6c.

36 Message du Conseil fédéral du 26 juin 1996 relatif à l'initiative populaire « pour la protection de l'être humain contre les techniques de reproduction artificielle (Initiative pour une procréation respectant la dignité humaine, PPD) » et à la loi fédérale sur la procréation médicalement assistée (LPMA), FF 1996243 s. Voir également Message du Conseil fédéral du 29 novembre 2002 relatif à la loi fédérale sur le partenariat enregistré entre personnes du même sexe, FF 20031222.

37 Message du Conseil fédéral du 26 juin 1996 relatif à l'initiative populaire « pour la protection de l'être humain contre les techniques de reproduction artificielle (Initiative pour une procréation respectant la dignité humaine, PPD) » et à la loi fédérale sur la procréation médicalement assistée (LPMA), FF 1996244.

38 Commission nationale d'éthique, La procréation médicalement assistée. Considérations éthiques et propositions pour l'avenir, Prise de position $n^{\circ} 22 / 2013,9$. 
son partenaire de même sexe, soit, en d'autres termes, qu'un enfant ait deux pères ou deux mères (cf. art. 264c CC).

[25] L'intérêt de l'enfant constitue bien entendu un but légitime (cf. art. 3 al. $1 \mathrm{CDE}^{40}$ ), mais les moyens invoqués par le Conseil fédéral et le TF pour garantir sa prise en compte ne sont pas appropriés et, partant, sont inadmissibles. S'il faut admettre, comme l'ont relevé Belser/Jungo, que la stabilité du couple est un facteur déterminant pour garantir le bien-être de l'enfant, il n'en demeure pas moins que cette stabilité n'est pas nécessairement garantie par l'institution du mariage $^{41}$. Ce constat avait d'ailleurs déjà été tiré par la CourEDH en 2007 dans l'affaire Emonet et autres c. Suisse ${ }^{42}$, mais n'a néanmoins pas incité le législateur suisse, dans le cadre de la révision du droit de l'adoption, à étendre le bénéfice de l'adoption conjointe aux couples hétérosexuels concubins vivant en ménage commun depuis au moins trois ans (art. 264a al. 1 CC a contrario), cela de manière à éviter de devoir accorder ce droit aux couples de même sexe sous peine de créer un régime discriminatoire ${ }^{43}$. Dans le même sens, le critère de la stabilité - dont l'institution du mariage serait garante - ne permet pas de justifier l'exclusion des couples de même sexe. En effet, comme l'a fait valoir la Cour constitutionnelle autrichienne, il ne fait pas de doute que ces couples sont en mesure d'avoir des relations stables dès lors qu'ils peuvent, selon la jurisprudence de la Cour européenne des droits de l'homme, invoquer la protection de leur vie familiale ${ }^{44}$.

[26] En résumé, au vu des développements de la Cour dans l'affaire Taddeucci et McCall c. Italie, il paraît possible de soutenir que la réglementation de l'art. 3 al. 3 LPMA est discriminatoire, en ce sens que l'exigence du statut marital constitue un obstacle insurmontable pour les couples de même sexe et qu'aucun motif objectif n'est susceptible de le justifier.

[27] Avant de passer à l'examen du projet de mariage pour tous et toutes, rappelons que d'autres auteures sont arrivées au même constat, mais selon une argumentation différente. BELSER/JUNGO ont en effet proposé un raisonnement en deux temps : selon elles, il s'agit, en premier lieu, de faire constater la non-conventionalité de la limitation du don de sperme aux couples hétérosexuels mariés - par opposition aux couples hétérosexuels non mariés. Comme relevé précédemment, l'objectif de l'art. 3 al. 3 LPMA n'étant pas tant de garantir la position particulière du mariage dans l'ordre juridique ${ }^{45}$ que d'assurer à l'enfant une certaine stabilité, l'exigence du statut marital ne constitue pas un motif apte à justifier l'exclusion des concubins hétérosexuels du don de sperme. À cet égard, le critère de durée du couple devrait suffire à permettre d'accéder au don de sperme. Une fois la non-conventionnalité de l'exigence du mariage pour les couples hétérosexuels reconnue, il s'agira de démontrer que la différence de traitement entre les couples hétérosexuels

40 Convention relative aux droits de l'enfant du 20 novembre 1989, RS 0.107.

41 Commission nationale d'éthique, La procréation médicalement assistée. Considérations éthiques et propositions pour l'avenir, Prise de position $n^{\circ} 22 / 2013$, p. 19.

42 «[... ] aux yeux de la Cour, l'argument du Gouvernement selon lequel l'institution du mariage garantit à la personne adoptée une stabilité accrue par rapport à l'adoption par un couple de concubins n'est plus forcément pertinent de nos jours ", CourEDH, Emonet et autres c. Suisse, 13 décembre 2007, n 39051/03, § 81.

43 Message du Conseil fédéral du 28 novembre concernant la modification du code civil (Droit de l'adoption), FF 2015872.

44 Verfassungsgerichtshof (VfGH), Erkenntnis 10.12.2013 G 16/2013-16, G 44/2013-14, § 2.1. Rappelons que dans cet arrêt, la Cour a jugé inconstitutionnelle l'exclusion des couples de femmes du don de sperme.

45 Ce qui est encore admis par la Cour européenne, CourEDH, X. c. Autriche (GC), 19 février 2013, n 19010/07, $\S 106$. 
non mariés et les couples de femmes n'est pas admissible ${ }^{46}$ dès lors que la différence se fonde sur le seul critère de l'orientation sexuelle et ne peut se justifier par le bien de l'enfant.

\section{Projet de mariage pour tous et toutes}

[28] Cette conclusion nous amène à l'examen du projet de mariage pour tous et toutes actuellement pendant devant les Chambres fédérales. Le Parlement a examiné différentes variantes dont l'une vise à simultanément proposer l'ouverture de l'institution du mariage aux couples de même sexe et l'accès au don de sperme aux couples de femmes mariées. Comme évoqué, cette variante n'est, à ce jour, soutenue ni par la Commission des affaires juridiques du Conseil national, ni par le Conseil fédéral, au motif qu'il s'agit " de ne pas surcharger le projet ${ }^{47}$. À cela s'ajouteraient "les objections soulevées lors de la consultation contre la présomption de maternité de l'épouse de la mère ", notamment au regard du droit de l'enfant de connaître ses origines lorsque le don de sperme a eu lieu à l'étranger de manière anonyme ou lorsque la conception s'est faite de manière naturelle ${ }^{48}$. Se pose dès lors la question de savoir si un report de l'ouverture du don de sperme aux couples de femmes est admissible au regard de notre analyse préalable.

[29] Nous avons vu que la notion de stérilité consacrée tant par la Constitution fédérale (art. 119 al. 2 let. c Cst. féd.) que par la LPMA (art. 5 LPMA) ne permet pas d'exclure les couples de même sexe. En d'autres termes, si le mariage pour tous et toutes devait être adopté, le critère du mariage ne serait plus pertinent, et l'exclusion des couples de femmes de l'accès à la PMA se fonderait alors sur leur seule orientation sexuelle : bien que mariés, les couples de femmes n'auraient pas, contrairement aux couples hétérosexuels, accès au don de sperme.

[30] Une telle différence de traitement serait-elle alors susceptible de se justifier par un but légitime et proportionné?

[31] S'il ne fait pas de doute que l'objectif « de ne pas surcharger le projet » n'est absolument pas susceptible d'entrer en considération, qu'en est-il des objections liées à la présomption de maternité de l'épouse de la mère mises en avant par le Conseil fédéral ? Pour rappel, une telle présomption soulèverait, selon le Conseil fédéral, « la question de la compatibilité entre [celle-ci] et le droit de l'enfant à connaître son ascendance, garanti par la Constitution, lorsque le don de sperme a eu lieu dans un pays où il peut être anonyme, ou quand la conception est naturelle ${ }^{49}$. [32] S'agissant du premier point, il n'est pas convaincant. Bien au contraire, comme déjà relevé à maintes reprises par la doctrine, c'est l'exclusion des couples de femmes du don de sperme qui est susceptible de poser problème au regard du droit de l'enfant de connaître ses origines. Dans ces conditions, les couples de femmes se voient en effet contraints de se rendre à l'étranger pour avoir accès au don de sperme, qui est parfois proposé de manière anonyme. Une légalisation permettrait donc d'éviter cette nécessité de se rendre à l'étranger et garantirait par là-même le droit de l'enfant de connaître son ascendance biologique (cf. art. 119 al. 2 let. g Cst. féd. ${ }^{50}$.

46 Belser/Jungo (note 10), p. 203.

47 Avis du Conseil fédéral du 29 janvier 2020, Initiative parlementaire " Mariage civil pour tous », FF 20201226.

48 Avis du Conseil fédéral du 29 janvier 2020, Initiative parlementaire " Mariage civil pour tous », FF 20201226.

49 Avis du Conseil fédéral du 29 janvier 2020, Initiative parlementaire " Mariage civil pour tous », FF 20201226.

50 Valérie Junod/Dorothea Wunder/Samia Hurst, Procréation médicalement assistée \& Préservation de la fertilité, in : Jusletter 27 août 2018, Rz 22 et les références citées ; LiSA MARGOT, Le droit à la connaissance des origines de l'enfant né de procréation médicalement assistée, FamPra 2017, p. 718 ss. 
[33] S'agissant des difficultés liées à la présomption de maternité en cas de conception naturelle, elles ne devraient pas poser plus de problèmes que dans le cas des couples hétérosexuels. Il ressort en effet clairement de l'art. 23 LPMA que l'exclusion des actions juridiques contre le donneur ne vaut qu'en cas de PMA. En cas de conception naturelle avec le sperme d'un donneur, les dispositions générales du droit civil s'appliquent, et il n'est dès lors pas exclu qu'un lien de filiation juridique soit établi à l'égard du donneur ${ }^{51}$. Si tel est le cas à l'égard du père d'intention, tel devra également être le cas à l'égard de la mère d'intention. Pour le surplus, il y a lieu de rappeler que de nombreux pays ont déjà apporté des réponses à cette question et qu'il existe donc de nombreuses sources d'inspiration pour le législateur ${ }^{52}$.

[34] Avant de conclure, relevons encore que si le législateur devait entériner son refus d'ouvrir le don de sperme aux couples de femmes mariées, ceux-ci n'auront alors d'autre choix que de continuer à recourir à un don de sperme à l'étranger. Or, à leur retour en Suisse, ces couples de femmes se verront discriminés par rapport aux couples hétérosexuels mariés dès lors que, contrairement à ces derniers, pour lesquels le lien de filiation à l'égard du père d'intention est présumé (cf. art. 252 al. 2, 255 al. 1 CC et 23 LPMA), ils devront passer par une procédure d'adoption ${ }^{53}$.

\section{Conclusion}

[35] En conclusion, nous sommes donc d'avis que la situation actuelle, qui réserve le don de sperme aux couples hétérosexuels mariés, est critiquable au regard de la jurisprudence récente de la Cour européenne des droits de l'homme. L'exigence du statut marital, consacrée par l'art. 3 al. 3 LPMA, constitue en effet un obstacle insurmontable pour les couples de même sexe qui n'est susceptible de se justifier par aucun motif objectif.

[36] Au vu de ce premier constat, il ne fait donc pas de doute que si le projet de mariage pour tous et toutes devait être adopté, il devrait alors nécessairement être accompagné d'une ouverture du don de sperme aux couples de femmes.

[37] Rappelons, pour conclure, que la situation actuelle est également particulièrement critiquable du point de vue des enfants ${ }^{54}$. Dès lors que les couples de femmes doivent se rendre à l'étranger pour pratiquer une PMA et recourir ensuite à une procédure d'adoption en Suisse pour établir un lien de filiation à l'égard de la seconde mère, les enfants nés dans ce cadre sont discriminés par rapport aux enfants nés d'une PMA dans le cadre d'un mariage hétérosexuel, dont le lien de filiation à l'égard de leur père $\mathrm{d}^{\prime}$ 'intention est automatiquement établi à la naissance et le droit de connaître leurs origines garanti.

Véronique Boillet est professeure de droit constitutionnel au Centre de droit public de l'Université de Lausanne.

\footnotetext{
Commission nationale d'éthique, Le don de sperme, Prise de position n 32, Berne 2019, p. 14.

Voir par ex. Guillaume Kessler, Regards comparatistes sur la PMA « pour toutes », AJ Famille 2020 p. 117 ss.

3 Dans le même sens, Loveday Hodson, Towards a Child-Centred Approach to Lesbian, Gay, Bi-Sexual and Transgender Families under the ECHR, International Journal of Children's Rights 20/2012, p. 514 ss. 